\title{
Spezialaktion für 50- bis 65-Jährige
}

\section{Neue, innovative und sichere Kapitalanlage}

Bei einer Anlage wird man normalerweise vor die Wahl gestellt: Sicherheit oder Rendite? Bei der innovativen Lösung Helvetia Value Trend erhalten Sie jedoch beides. Die Helvetia garantiert Ihnen nach 10 Jahren die Rückzahlung Ihres Kapitals und eines Mindestzinses. Während der Laufzeit profitieren Sie mit Ihrem Kapital zusätzlich von einer Index-Strategie, welche die wichtigsten Märkte wie Aktien, Obligationen und Rohstoffe abdeckt. Liegt die Wertentwicklung zum Ende der Laufzeit über der garantierten Auszahlung, erhalten Sie diesen höheren Wert ausbezahlt.

\section{Steuerfreie Auszahlung}

Von der steuerfreien Auszahlung profitieren alle Kunden, welche am 12. Dezember 2011 50- bis 65-jährig sind.

\section{Berechnungsbeispiel: Frau, 55-jährig, Investition CHF 100000.-}

\begin{tabular}{|l|l|}
\hline Garantierte Auszahlung & CHF 111026.- \\
\hline Auszahlung bei 3\% p. a. * & CHF 125386.- \\
\hline Auszahlung bei 4\% p. a. * & CHF 138106.- \\
\hline Auszahlung bei $5 \%$ p. a. * & CHF 151974.- \\
\hline
\end{tabular}

* nicht garantierte Wertentwicklung der Index-Strategie

\section{Das Wichtigste auf einen Blick}

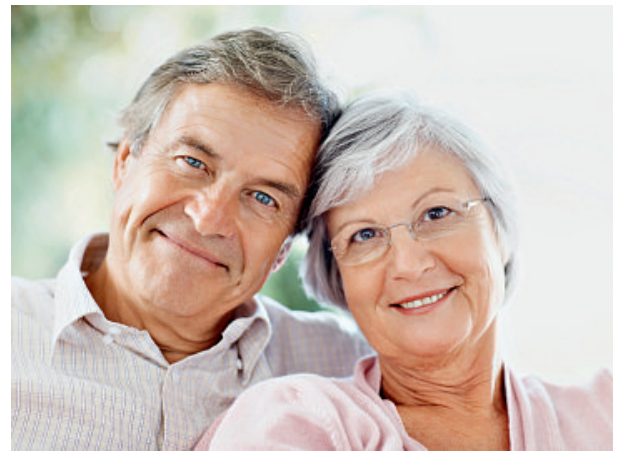

$\rightarrow$ Helvetia Value Trend ist zeitlich und betraglich limitiert und bis spätestens am 9.12.2011 erhältlich.

$\rightarrow$ Die Vertragsdauer beträgt 10 Jahre.

$\rightarrow$ Vertragsbeginn ist der 12. Dezember 2011, wobei vorzeitige Zahlungen mit $2 \%$ verzinst werden.

$\rightarrow$ Die Stempelsteuer von 2,5\% erhalten Sie geschenkt!

$\rightarrow$ Sie können nur gewinnen!

Bestellen Sie noch heute Ihre persönliche Offerte und weitere Informationen zu dieser innovativen Lösung.

\section{Antworttalon}

Vorname / Name

Adresse

PLZ / Ort

Geburtsdatum

Telefon Privat / Geschäft

Beste Zeit für einen Anruf

E-Mail-Adresse

Investition

\section{$\mathrm{CHF}$}

Bitte senden Sie mir eine Offerte von Helvetia Value Trend.

$\bigcirc \quad$ Ich wünsche eine persönliche Beratung.

Bitte rufen Sie mich an.

Ich interessiere mich für:

Krankenkasse

Säule 3a

Finanz-/Steuerplanung
Pensionskasse BVG

Rechtsschutzversicherung

Berufshaftpflichtversicherung

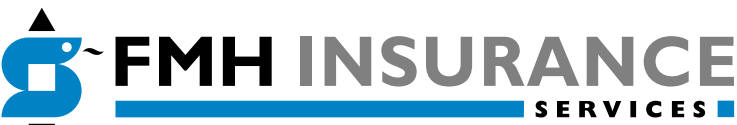

Roth Gygax \& Partner AG \& Koordinationsstelle Moosstrasse 2 @ 3073 Gümligen

Telefon 0319595000 a Fax 0319595010 mail@fmhinsurance.ch — www.fmhinsurance.ch 